\title{
Cariostatic Efficacy of Aqueous and Ethanolic Extracts of Liquorice in the Schoolchildren: In Vivo Comparative Study
}

\author{
Avanindra Kumar ${ }^{1}$, Tanoj Kumar ${ }^{2}$, Abhinav Jha ${ }^{3}$, Jay Kishore ${ }^{4}$, Akash D Barua ${ }^{5}$, Priyadershini Rangari ${ }^{6}$
}

\begin{abstract}
Aim: The present study was done to evaluate the in vivo cariostatic efficacy of aqueous and ethanolic extracts of liquorice to ascertain whether it could be developed into a caries-preventive regimen basically targeted for use in the pediatric population.

Materials and methods: Thirty schoolchildren of 6-12-year-old were selected for the study. Powder of Glycyrrhiza glabra is used to prepare the gel with various concentration of aqueous and ethanolic liquorice gel. The preweighed dose was delivered through the vials. The drug concentrations were based on their respective minimum bactericidal concentration (MBC) values against Streptococcus mutans, which were calculated earlier. And it is divided into three groups, i.e., group I: aqueous liquorice extract $1.75 \mathrm{~g} / 10 \mathrm{~mL}$ saline, group II: ethanolic liquorice extract $350 \mathrm{mg} / 10 \mathrm{~mL}$, and group III: hexidine $(0.2 \%$ chlorhexidine, CHX). For statistical analysis, Tukey's post hoc with one-way analysis of variance (ANOVA) and $t$ test were applied.

Results: It was found that hexigel has a potential antibacterial activity against S. mutans, with minimum inhibitory concentration (MIC) of 3.14 \pm 2.02 . Ethanolic liquorice shows MIC of $2.15 \pm 0.91$ and aqueous liquorice shows MIC of $1.30 \pm 1.08$. Tukey's post hoc test showed statistically nonsignificant difference between hexigel and ethanolic liquorice against $S$. mutans.

Conclusion: On conclusion, the present study found that hexigel was better than both the ethanolic and aqueous solutions of liquorice. And ethanolic liquorice was found to be better than aqueous solution, but it was not statistically significant, which could be due to the small sample size. Clinical significance: Dental caries is one of the most common infectious microbial diseases. Various steps have been taken to prevent dental caries, fluoride being the most common among them. Nowadays, G. glabra, commonly known as liquorice (mulethi), is one such medicinal plant used by various cultures for thousands of years to relieve coughs, sore throats, and gastric inflammation. This drug in our study demonstrated inhibitory effect on the growth of S. mutans.

Keywords: Antimicrobials, Glycyrrhiza glabra, Hexigel, Liquorice gel, Streptococcus mutans.

The Journal of Contemporary Dental Practice (2020): 10.5005/jp-journals-10024-2792
\end{abstract}

\section{INTRODUCTION}

Dental caries is one of the most common and widespread persistent diseases. It involves multifactorial, chronic, infectious, and transmissible and a dynamic process. The pathogenesis of this disease consists of bacterial adherence to dental enamel surfaces leading to the formation of colonies of dental plaque. This process further results in localized demineralization of tooth enamel by bacterial toxins and acids produced by the fermentation of dietary carbohydrates. ${ }^{1}$ The burden on the public health economy weighs down more by dental caries as it is a diseases that affects people of all age-groups, and it also leads to poor development and health parameters of the growing children. As prevention is always important, the cure usually requires more financial burden. Hence, the preventive measures along with the therapeutic protocol to minimize the effect of cariostatic bacteria are important.

It was Miller who postulated the chemicoparasitic theory for the etiology of dental caries. After a century of research, it has now been established that Streptococcus mutans are associated with caries in humans. The saliva contains $\geq 10^{6}$ S. mutans $/ \mathrm{mL}$, which poses a very high caries risk. ${ }^{2}$ Many studies have shown that S. mutans usually exceeded $30 \%$ of the cultivable plaque flora. ${ }^{3-5}$ These high-concentration bacteremia in dental infection is shown to be associated with carious lesions, white spot lesions, and sound tooth surfaces near the lesions.
1,2Department of Oral Pathology and Microbiology, Patna Dental College and Hospital, Patna, Bihar, India

${ }^{3}$ Department of Oral Pathology and Microbiology, Sarjug Dental College and Hospital, Darbhanga, Bihar, India

${ }^{4}$ Department of Dentistry, Primary Health Centre, Bihar Government, Vaishali, Bihar, India

${ }^{5}$ Department of Conservative Dentistry and Endodontics, Government Dental College and Hospital, Silchar, Assam, India

${ }^{6}$ Department of Dentistry, Sri Shankaracharya Institute of Medical Sciences, Bhilai, Durg (Chhattisgarh), India

Corresponding Author: Tanoj Kumar, Department of Oral Pathology and Microbiology, Patna Dental College and Hospital, Patna, Bihar, India, Phone: +91 7999449364, e-mail: panhealth121013@gmail.com

How to cite this article: Kumar A, KumarT, Jha A, et al. Cariostatic Efficacy of Aqueous and Ethanolic Extracts of Liquorice in the Schoolchildren: In Vivo Comparative Study. J Contemp Dent Pract 2020;21(5):575-579.

Source of support: Nil

Conflict of interest: None

However, the concentration of S. mutans is less than $0.1 \%$ of the plaque flora in individuals with negligible to no caries activity. ${ }^{6}$ Hence, it is clearly indicated that dental caries is an infectious disease and that $S$. mutans is one of the main infectious agents; few

() The Author(s). 2020 Open Access This article is distributed under the terms of the Creative Commons Attribution 4.0 International License (https://creativecommons. org/licenses/by-nc/4.0/), which permits unrestricted use, distribution, and non-commercial reproduction in any medium, provided you give appropriate credit to the original author(s) and the source, provide a link to the Creative Commons license, and indicate if changes were made. The Creative Commons Public Domain Dedication waiver (http://creativecommons.org/publicdomain/zero/1.0/) applies to the data made available in this article, unless otherwise stated. 
studies $^{7-9}$ also emphasize the role of diet in the clinical expression of this infection causing enamel destruction.

Recently it was observed that there is a spike in the use of plant extracts in modern medicine, which could be attributed to the search for effective, inexpensive, innocuous, and efficient alternatives. This led to the use of natural phytochemicals resulting from plants for curing diseases of the human body. One such herb is liquorice root. It is known to be used in Chinese medicine and Ayurveda for centuries. Liquorice belongs to the Glycyrrhiza species which is native to Mediterranean countries and Asia. The term Glycyrrhiza was coined from the ancient Greek words: "glycos" meaning sweet and "rhiza" meaning root. ${ }^{10}$ About 30 species are classified under the genus Glycyrrhiza, though the dried and skinned roots of G. glabra Linn. and G. uralensis Fisch. (Fam. Leguminosae) are the commonest sources of liquorice.

The rich secondary metabolites of liquorice have been related with various health benefits. It has shown to have a beneficial effect in the treatment of various diseases such as cancer, tuberculosis, atherosclerosis, gastric ulcers, immunodeficiency, hepatitis, and bacterial infections. ${ }^{11-14}$

The United States Food and Drug Administration (FDA) has generally recognized liquorice as safe. Hence, it is considered safe for human consumption as long as it is consumed in small amounts and by individuals who are not sensitive to glycyrrhizin. ${ }^{10}$

The anticariogenic properties of liquorice have been studied for many years; however, only a few studies assessing its anticariogenic role have been published. Pterocarpenes, namely, glycyrrhizol $A$ and glycyrrhizol B and four other known isoflavonoids, namely, 5-O-methylglycryol, isoglycyrol, 6,8-diisoprenyl-5,7,40trihydroxyisoflavone, and gancaonin $\mathrm{G}$ have been extracted by $\mathrm{He}$ et al. from the roots of $G$. uralensis, and it was observed that all these metabolites show activity against $S$. mutans, with glycyrrhizol $A$ and 6,8-diisopreny 1-5,7,40-trihydroxyisoflavone showing the highest antimicrobial activity against these bacteria. ${ }^{15}$

As the present pharmacological products contain various hazardous chemicals and carcinogenic compositions, it is necessary to discover the safer regimen for prevention of dental caries in pediatric population. The present study was done to evaluate the in vivo cariostatic efficacy of aqueous and ethanolic extracts of liquorice to ascertain whether it could be developed into a caries-preventive regimen basically targeted for use in the pediatric population.

\section{Materials and Methods}

A randomized controlled trial in the Department of Endodontics, Government Dental College, Patna (Bihar) was conducted from June 2019 to November 2019. Thirty children in the age-group of 6-12, with an informed written consent were included in this study. Children having at least $5 \mathrm{D}$ in the decayed missed and filled surfaces (DMFS) score, which implies the caries' active subject, who have not taken any antibiotic for the last 6 months, and are otherwise healthy with no infection other than caries were included in this study. Patients undergoing any dental treatment, which had received any antibiotic therapy within the past 6 months and/or are under any oral hygiene regimen including mouthwash, were excluded from the study.

\section{Preparation of Liquorice Extract and Calculation Methods for MIC and MBC}

For preparation of liquorice extract, the liquorice roots were obtained from Haryana where it is widely cultivated. These roots were then dried in shade and ground in an electric grinder. The powder was then divided in to two parts, one of which was soaked in distilled water and the other in ethyl alcohol for 24 hours. After 24 hours, both the mixtures were filtered using muslin cloth and Whatman no. 1 filter paper. Filtrate from each extract was then lyophilized and stored in a refrigerator at $4^{\circ} \mathrm{C}$ for further use. Powder of $G$. glabra was used to prepare the gel with various concentrations of aqueous and ethanolic liquorice extracts. The gel preparation was done in the laboratory of pharmacology department of the same institute by the principal investigator.

To evaluate the antimicrobial efficacy and toxicity of liquorice extracts, we calculated the minimum inhibitory concentration (MIC) and minimum bactericidal concentration (MBC) against $S$. mutans for both the aqueous and ethanolic extracts using the broth tube dilution method (macro dilution). ${ }^{7}$ Isolation of S. mutans strain was done from the saliva samples of patients having high caries activity. For culturing selective medium, mitis salivarius-bacitracin agar was used. ${ }^{17}$ Colonies of S. mutans were pale blue, granular, and had frosted glass appearance.

Calculation of MIC is done by macro broth dilution method. For calculation of MIC, $10 \mathrm{~mL}$ test tubes were taken and brain heart infusion broth was added to each of these $10 \mathrm{~mL}$ test tubes, an equal volume of $S$. mutans adjusted to $0.5 \mathrm{McFarland}$ was then added to these test tubes. Minimum inhibitory concentration was then detected by visual inspection. The lowest concentration without any visual turbidity of the test agent with clear supernatant was considered as the MIC.

To calculate MIC for the alcoholic licorice root extract, a solution of $50 \%$ concentration was prepared as the stock solution. To achieve the working concentration, a serial dilution 50\%, $25 \%$, $12.5 \%, 6.25 \%, 3.1 \%, 1.56 \%, 0.78 \%, 0.39 \%, 0.19 \%$, and $0.09 \%$ of the extract was prepared. ${ }^{16} \mathrm{~A}$ single examiner conducted all the measurements for the zone of inhibition. Prior to and during the study calibration of examiner was done by reexamining $5 \%$ of the samples; this was done to minimize the intraexaminer variability. To meet the scientific requirement for validity and reliability, intraexaminer agreement was determined using kappa statistics $(\kappa)$, according to Landis and Koch. A similar procedure was done to test the antimicrobial effect of the alcoholic liquorice root extract.

For calculation of MBC, a small amounts was taken from all the tubes with no visible bacterial growth and seeded into Mueller-Hinton agar and incubated overnight at $37^{\circ} \mathrm{C}$. Minimum bactericidal concentration was inferred to be the concentration at which no colonies of $S$. mutans appeared. The major constituents of lyophilized plant extracts of liquorice were analyzed by using standard qualitative methods. ${ }^{18,19}$

\section{Collection of Sample}

All the subjects participating in the study were asked not to brush their teeth on the day of sampling and follow their normal daily diet intake. Unstimulated salivary samples were then collected after at least 2 hours of meal. Subjects were then divided into three groups (Figs 1 and 2).

- Group I: Patients receiving aqueous liquorice gel,

- Group II: Patients receiving ethanolic liquorice gel, and

- Group III: Patients received a commercially available CHX mouthwash (hexidine).The preweighed dose was delivered through the vials. The drug concentrations were based on their respective $M B C$ values against $S$. mutans which were calculated earlier. 


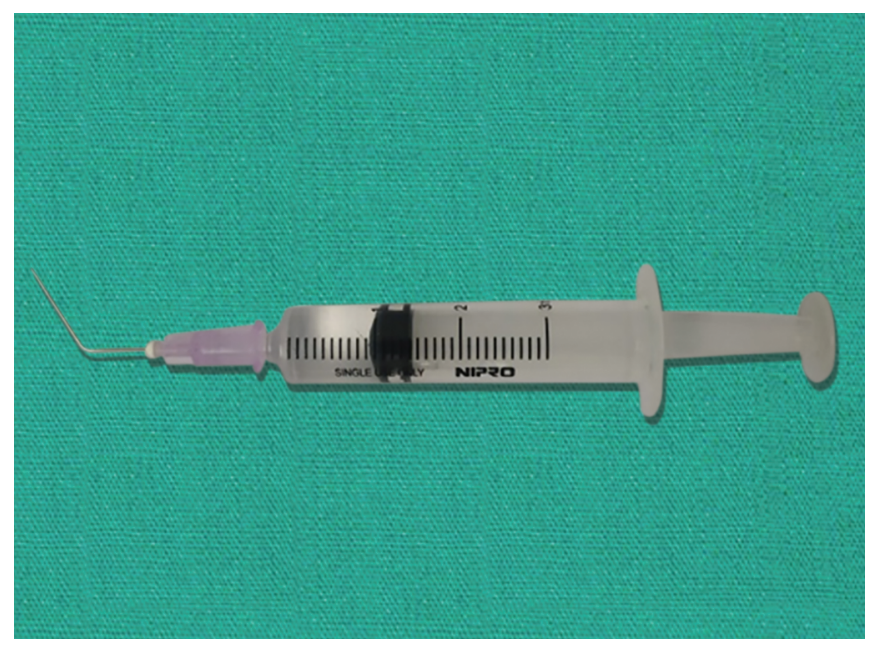

Fig. 1: Syringe loaded with alcoholic liquorice

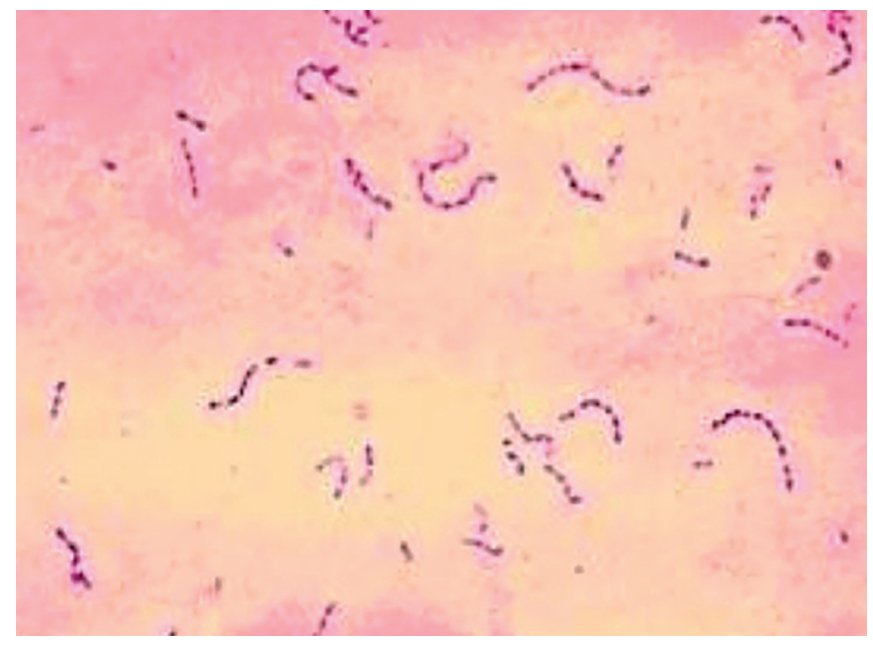

Fig. 3: Microscopic image of Streptococcus mutans bacteria

- Group I: aqueous liquorice extract $1.75 \mathrm{~g} / 10 \mathrm{~mL}$ saline,

- Group Il: ethanolic liquorice extract $350 \mathrm{mg} / 10 \mathrm{~mL}$,

- Group III: hexidine (0.2\% CHX).

The subjects were asked to rinse with $10 \mathrm{~mL}$ of their respective solutions for 1 minute. To collect the unstimulated saliva, subjects were asked to let saliva pool in their mouth for 2 minutes and then collect the accumulated saliva into sterile, labeled saliva-collecting cups. Four saliva samples were collected from each patient. One prerinse and three postrinse samples were collected immediately, 15 minutes, and 30 minutes after the oral rinse. The $\mathrm{pH}$ of the unstimulated whole saliva was also collected at each interval and was analyzed using a chair side kit (GC saliva check). The $\mathrm{pH}$ test paper was immersed in the sample for 10 seconds and the color changes were compared with the proprietary chart provided by the manufacturer.

For counting of the $S$. mutans colony, the collected saliva cups were taken in refrigerated box to the microbiological department of the institute. The vortexes saliva samples were then diluted in a 1:50 ratio with normal saline. Aliquot $(0.004 \mathrm{~mL})$ of mixed, uncentrifuged, diluted saliva which was spread over a plate of selective media for S. mutans, i.e., mitis salivarius-bacitracin agar with nichrome SWG 28 wire loop. All the plates were incubated at $37^{\circ} \mathrm{C}$ in a micro-aerophilic

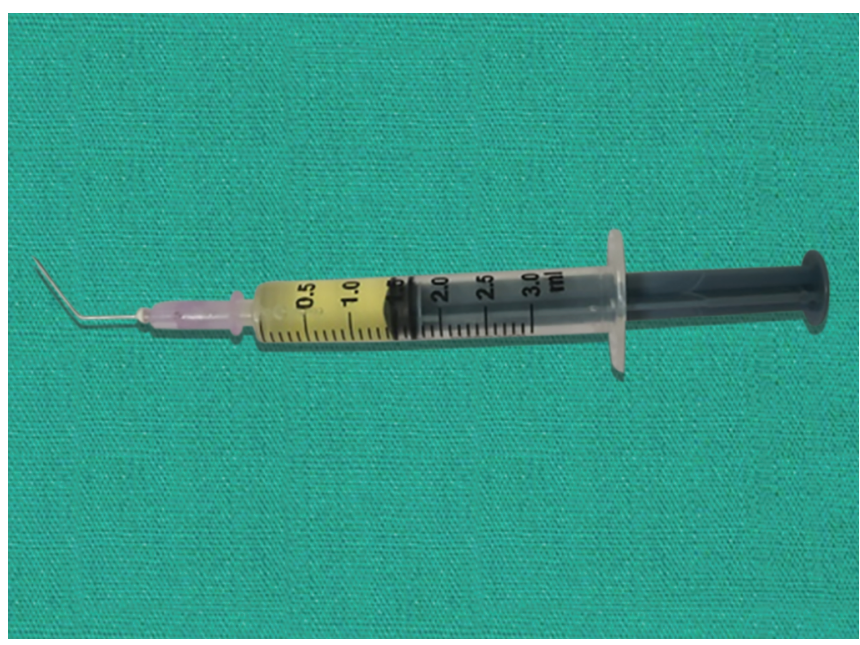

Fig. 2: Syringe loaded with aqueous liquorice

Table 1: Minimum inhibitory concentration and minimum bactericidal concentration of hexigel, ethanolic, and aqueous liquorice extracts

\begin{tabular}{lll}
\hline Solutions tested & MIC & MBC \\
\hline Hexigel & $3.14 \pm 2.02$ & $3.25 \pm 2.01$ \\
Ethanolic liquorice extract & $2.15 \pm 0.91$ & $2.26 \pm 0.89$ \\
Aqueous liquorice extract & $1.30 \pm 1.08$ & $1.40 \pm 1.19$ \\
ANOVA $(F)$ & 12.56 & 12.34 \\
$p$ value & 0.000 & 0.000 \\
\hline
\end{tabular}

Tukey honestly significant difference (HSD) post hoc test for MIC

environment at $5 \% \mathrm{CO}_{2}$ for 48 hours. The colony counts for $S$. mutans were estimated by a semiquantitative method, i.e., standard loop method as described by Collee et al. ${ }^{20}$

The identified colonies were counted using a digital colony counter. The confirmation of S. mutans was done with the help of light microscope after staining a heat-fixed smear slide (Fig. 3). The intergroup comparisons of the values of $\mathrm{pH}$ and colony counts were made at baseline and after the oral rinse. One-way analysis of variances (ANOVAs) with Tukey's post hoc and $t$ test are applied for statistical analysis.

\section{Results}

In the present study, the antibacterial activity of hexidine, G. glabra, in aqueous and ethanolic solutions and their potency were quantitatively and qualitatively assessed by determining MIC and $\mathrm{MBC}$ and the diameter of the inhibition zone. The MIC for aqueous and ethanolic extracts of liquorice and hexigel were 3.14, 2.15, and $1.30 \mathrm{MBC}$ for the same were $3.25,2.26$ and 1.40 respectively (Table 1). The serial dilution to achieve the working concentration for the extract was as described above.

Group I vs group II: Diff. $=-0.9900,95 \%$ confidence interval $(\mathrm{Cl})=-1.8661$ to $-0.1139, p=0.0228$

Group I vs group III: Diff. $=-1.8400,95 \% \mathrm{Cl}=-2.7161$ to -0.9639 $p=0.0000$

Group II vs group III: Diff. $=-0.8500,95 \% \mathrm{Cl}=-1.7261$ to 0.0261 , $p=0.0592$

Tukey HSD post hoc test MBC

Group I vs group II: Diff. $=-0.9900,95 \% \mathrm{Cl}=-1.8785$ to -0.1015 , $p=0.0252$ 
Table 2: Sex and mean age, mean DMF score, and frequency of daily toothbrushing

\begin{tabular}{|c|c|c|c|c|c|}
\hline & \multicolumn{2}{|c|}{ Sex } & \multirow[b]{2}{*}{ Age } & \multirow[b]{2}{*}{$D M F$} & \multirow{2}{*}{$\begin{array}{l}\text { Frequency of } \\
\text { toothbrushing }\end{array}$} \\
\hline & $M$ & $F$ & & & \\
\hline Hexigel & 6 & 4 & $8.8 \pm 1.6$ & $9 \pm 1.89$ & $1.3 \pm 0.45$ \\
\hline Ethanolic liquorice extract & 5 & 5 & $8.5 \pm 1.69$ & $8.7 \pm 1.62$ & $1.4 \pm 0.48$ \\
\hline Aqueous liquorice extract & 7 & 3 & $8.2 \pm 2.14$ & $8.8 \pm 1.54$ & $1.2 \pm 0.4$ \\
\hline
\end{tabular}

Group I vs group III: Diff. $=-1.8500,95 \% \mathrm{Cl}=-2.7385$ to -0.9615 , $p=0.0000$

Group II vs group III: Diff. $=-0.8600,95 \% \mathrm{Cl}=-1.7485$ to 0.0285 , $p=0.0600$

In this study of the solutions, we found that the highest MIC was that of hexigel followed by ethanolic extract of licorice. The difference between hexigel and ethanolic solution was significant, and the difference between aqueous and ethanolic solutions of licorice was also significant, which made the ethanolic solution better than the aqueous solution.

The variable characteristics of the three groups at admission are summarized in Table 2. The factors, viz., sex and mean age, mean decay missing filled (DMF) score, and the frequency of daily toothbrushing were recorded. It was found that the above-mentioned factors did not influence the study outcome measures and were comparable.

The mean S. mutans colony counts at baseline (pre-treatment) did not show significant difference between the three groups; hence, this signifies that all the three groups are comparable at the baseline. The mean colony counts in all three groups did not decrease significantly $(p \geq 0.001)$ immediately after rinsing (Table 3 ).

The reduction in colony count immediately postrinse was not significant in the control group, with the $p$ value of 0.714 calculated by one-way ANOVA test and Tukey HSD post hoc test with $95 \% \mathrm{Cl}$.

The reduction in the colony counts when compared with prerinse and after 15 minutes of rinse was statistically significant, with $p$ value 0.051 in the control group. While comparing pre- and 30 minutes postrinse, the $p$ value computed was 0.0175 which is considered to be significant. This means that hexigel as a commercial product is effective against S. mutans, and the effect is significant after 15 minutes of rinse which became more significant after 30 minutes of rinse.

\section{Discussion}

Even today, oral infections and dental caries are still a serious public health problem and levy a major liability to healthcare services all round the world and are more pronounced in developing countries.

Dental caries is one of the most common infectious microbial diseases. Various steps in have been taken to prevent dental caries, fluoride being the most common among them. It is now established that dental caries is an infectious disease; its etiology demonstrates microorganism as the major causative factor, $S$. mutans being highly associated with it, hence, altering the host response to this microorganism may lead to the prevention of this disease. The growing cause of concern of these days is the development of resistance to antibiotics and antiseptics, thus, limiting the preventive measures. Antimicrobial activity of plant products has been studied in different regions of the world, and G. glabra, commonly called as licorice, is one such product.

Glycyrrhiza glabra, commonly known as liquorice (mulethi), is a medicinal plant used by various tribal cultures for thousands of years to relieve cough, sore throats, and gastric inflammation.
In our study, we tested the antibacterial activity of this plant product. We first calculated the MIC and MBC of G. glabra in ethanolic and aqueous solutions and compared it with a commercially available $\mathrm{CHX}$ product (hexigel), which we used as a control.

In our study, the antibacterial activity of G. glabra in vitro and their potency were assessed quantitatively and qualitatively by determining the zone diameter of inhibition, MIC, and MBC as shown in Table 1.

In our study, for hexigel, both MIC and MBC were computed to be 3.14 and 3.25, respectively. In a study conducted by Sedighinia et al., ${ }^{21}$ the MIC for CHX was found to be 0.0625 and MBC 0.125 ; this difference may be due to the different commercial compounds used and different biological habitat as their study was conducted in Iran.

In our study, the MIC and MBC for ethanolic compounds were 2.15 and 2.26, respectively. In a study conducted by Sedighinia et al., ${ }^{21}$ both the MIC and MBC were calculated as 12.5, which could be due to the different techniques used and different concentrations of the solution prepared.

Hence, the MIC and MBC of hexigel was better than ethanolic or aqueous preparation of liquorice. While comparing ethanolic liquorice and hexigel, we found that hexigel was significantly superior to ethanolic liquorice, with a $p$ value of 0.02 . The difference between aqueous liquorice and hexigel was also significant with $p$ value of 0.000 . While comparing both ethanolic and aqueous liquorice, ethanolic liquorice was superior, with $p$ value of 0.059 . These results are in accordance with another study conducted by Nirmala and Selvaraj ${ }^{22}$ In another study conducted by Sedighinia et al., ${ }^{21} \mathrm{CHX}$ was slightly better than ethanolic and aqueous liquorice but not statistically significant.

In the in vivo study conducted by the authors of this study, we asked our patients to rinse with the given solutions and took the sample just before the rinse, immediately after rinse, 15 minutes after rinse, and 30 minutes after rinse. The colonies were then cultured by the technique explained above and colony counting of S. mutans was done. We found that hexigel was better than both liquorice solutions but the difference was not statistically significant, though in the in vitro study the MIC and MBC of hexigel were significantly better than the other two solutions. This may be due to the difference in the natural variable factors that were absent in the in vitro study (Table 3). The mean inhibition rates were more with alcoholic licorice root extract compared to that in the aqueous form, which could be due to the better solubility of the liquorice compound in alcohol or the very presence of alcohol itself. Our results are in consistence with the study conducted by Nirmala and Selvaraj, ${ }^{22}$ but in the study conducted by Ajagannanavar et al., ${ }^{23}$ they found that alcoholic licorice root extract shows superior inhibitory effect against S. mutans and Lactobacillus acidophilus as compared to the aqueous form and CHX. This may be due to the in vitro nature of the study.

It is difficult to compare our results with the in vivo studies reported in literature because of the difference in methodology and preparation of liquorice solutions. 
Cariostatic Efficacy of Aqueous and Ethanolic Extracts of Liquorice

Table 3: Pre and postrinse S. mutants colony counts (CFU per $\mathrm{mL}$ of saliva) in three groups (values are mean)

\begin{tabular}{lllll}
\hline Solutions tested & Prerinse & $\begin{array}{l}\text { Immediately after } \\
\text { rinse }\end{array}$ & $\begin{array}{l}15 \text { minutes after } \\
\text { rinse }\end{array}$ & $\begin{array}{l}30 \text { minutes after } \\
\text { rinse }\end{array}$ \\
\hline Hexigel & $6.67 \pm 1.47$ & $6.53 \pm 1.48$ & $5.93 \pm 1.41$ & $5.77 \pm 1.38$ \\
Ethanolic liquorice extract & $6.8 \pm 1.9$ & $6.47 \pm 1.74$ & $6.30 \pm 1.74$ & $6.27 \pm 1.74$ \\
Aqueous liquorice extract & $6.8 \pm 1.97$ & $6.7 \pm 1.51$ & $6.67 \pm 1.45$ & $6.67 \pm 1.49$ \\
ANOVA $(F)$ & 0.0525 & 0.171 & 1.7309 & 2.55 \\
$p$ value & 0.9489 & 0.843 & 0.1832 & 0.083 \\
\hline
\end{tabular}

Study conducted by Segal et al. ${ }^{24}$ showed that licorice did not promote growth or induced plaque formation.

In a study conducted by Toors and Herczog, ${ }^{25}$ experimental licorice proved to be well fermentable by both S. mutans and the plaque-saliva mixture. They measured the acid production from an experimental, nonsugar licorice, in bacterial suspensions.

\section{Conclusion}

On conclusion, the present study found that hexigel was better than both the ethanolic and aqueous solutions of liquorice. And ethanolic liquorice was found to be better than aqueous solution, but it was not statistically significant, which could be due to the small sample size.

\section{References}

1. Mittal S, Hiregoudar M, Subramaniam R, et al. Dental effect of three herbal extracts against Streptococcus mutans and Lactobacillus acidophilus in comparison to chlorhexidine. J Indian Assoc Public Health Dent 2011;17:336-340.

2. Pallavi SK. Effect of chlorhexidine on Mutans streptococci and dental caries. J Indian Assoc Public Health Dent 2011;17:678-683.

3. Van Houte J, Gibbs G, Butera C. Oral flora of children with "nursing-bottle caries. J Dent Res 1982;61(2):382-385. DOI: 10.1177/00220345820610020201.

4. Berkowitz RJ, Turner J, Hughes C. Microbial characteristics of the human dental caries associated with prolonged bottle feeding. Arch Oral Biol 1984;29(11):949-951. DOI: 10.1016/0003-9969(84)90097-9.

5. Milnes AR, Bowden GH. The microflora associated with developing lesions of nursing caries. Caries Res 1985;19(4):289-297. DOI: 10.1159/000260858.

6. Loesche WJ. Role of streptococcus mutans in human dental decay. Microbiol Rev 1986;50(4):353-380. DOI: 10.1128/MMBR.50.4.353380.1986.

7. Keyes $P$. The infectious and transmissible nature of experimental dental caries. Arch Oral Biol 1960;1(4):304-320. DOI: 10.1016/00039969(60)90091-1.

8. Krasse B. The effect of caries inducing streptococci in hamsters fed diets with sucrose or glucose. Arch Oral Biol 1965;10(2):223-226. DOI: 10.1016/0003-9969(65)90023-3.

9. Dreizen S, Brown LR. Xerostomia and caries. In: Stiles HM, Loeche WJ, O'Brien T. Microbial aspects of dental caries. Washington DC: IRL; 1976. pp. 263-273.

10. Messier C, Epifano F, Genovese S, et al. Licorice and its potential beneficial effects in common oro-dental diseases. Oral Dis 2012;18(1):32e9. DOI: 10.1111/j.1601-0825.2011.01842.x.
11. Isbrucker RA, Burdock GA. Risk and safety assessment on the consumption of licorice root (Glycyrrhiza sp.), its extract and powder as a food ingredient, with emphasis on the pharmacology and toxicology of glycyrrhizin. Regul Toxicol Pharmacol 2006;26(3):167e92. DOI: 10.1016/j.yrtph.2006.06.002.

12. Shen XP, Xiao PG, Liu CX. Research and application of radix glycyrrhizae. Asian J Drug Metab Pharmacokinet 2007;7:181e200.

13. Nassiri-Asl M, Hosseinzadeh $\mathrm{H}$. Review of pharmacological effects of glycyrrhiza sp. and its bioactive compounds. Phytother Res 2008;22:709e24.

14. Kalani K, Chaturvedi V, Alam S, et al. Anti-tubercular agents from glycyrrhiza glabra. Curr Top Med Chem 2015;15(11):1043e9. DOI: 10. 2174/1568026615666150317223323.

15. He J, Chen L, Heber D, et al. Antibacterial compounds from glycyrrhiza uralensis. J Nat Prod 2006;69(1):121e4. DOI: 10.1021/np058069d.

16. National Committee for Clinical Laboratory Standards (NCCLS). Approved Standard M7-A5: methods for dilution antimicrobial susceptibility test for bacteria that grow aerobically. 5th ed., Wayne, PA: NCCLS; 2000

17. Gold OG, Jordan HV, Van Houte J. A selective medium for Streptococcus mutans. Arch Oral Biol 1973;18(11):1357-1364. DOI: 10.1016/0003-9969(73)90109-x.

18. Harborne JB. Phytochemicals Methods. London: Chapman and Hall Ltd.; 1973. pp. 49-188.

19. Trease GE, Evans WC. Pharmacognosy. 11th ed., London: Bailliere Tindall; 1989. pp. 45-50.

20. Collee JG, Duguid JP, Fraser AG, et al. Laboratory strategy in the diagnosis of infective syndromes. In: Collee JG, Fraser AG, Marmion $\mathrm{BP}$, et al. Mackie and McCartney practical medical microbiology. 14th ed., New York: Churchill Livingstone; 1996. pp. 53-94.

21. Sedighinia F, Safipour Afshar A, Soleimanpour S, et al. Antibacterial activity of Glycyrrhiza glabra against oral pathogens: an in vitro study. Avicenna J Phytomed 2012;2(3):118-124.

22. Nirmala P, Selvaraj T. Anti-inflammatory and anti-bacterial activities of lycyrrhiza glabra L. J Agr Tech 2011;7:815-823.

23. Ajagannanavar SL, Battur $\mathrm{H}$, Shamarao $\mathrm{S}$, et al. Effect of aqueous and alcoholic Licorice (glycyrrhiza glabra) root extract against Streptococcus mutans and Lactobacillus acidophilus in comparison to chlorhexidine: an in vitro study. J Int Oral Health 2014;6(4): 29-34.

24. Segal R, Pisanty S, Wormser R, et al. Anticariogenic activity of licorice and glycyrrhizine I: inhibition of in vitro plaque formation by Streptococcus mutans. J Pharm Sci 1985;74(1):79-81. DOI: 10.1002/ jps.2600740121.

25. Toors FA, Herczog Jl. Acid production from a nonsugar licorice and different sugar substitutes in Streptococcus mutans monoculture and pooled plaque-saliva mixtures. Caries Res 1978;12(1):60-68. DOI: 10.1159/000260316. 